\title{
OBJECT-BASED LAND COVER CLASSIFICATION FOR ALOS IMAGE COMBINING TM SPECTRAL INFORMATION
}

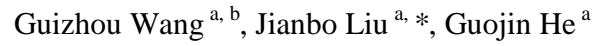 \\ ${ }^{\text {a }}$ Institute of Remote Sensing and Digital Earth, Chinese Academy of Sciences, Beijing, 100094, China - ( gzwang, jbliu, \\ gjhe )@ceode.ac.cn \\ ${ }^{\mathrm{b}}$ University of Chinese Academy of Sciences, Beijing, 100049, China
}

Commission IV, WG IV/4

KEY WORDS: Object-based classification, High spatial resolution remote sensing image, Spatial mapping mechanism

\begin{abstract}
:
Land cover classification for high spatial resolution remote sensing images becomes a challenging work. The high spatial resolution remote sensing images have more spatial information. The low or medium resolution remote sensing images have more spectral information. In order to improve the accuracy of high spatial resolution remote sensing image classification, additional information should be incorporated into the classification process of high spatial resolution remote sensing image. This paper proposed a method of object-based land cover classification for high spatial resolution ALOS images combining the spectral information of TM images. First, the high spatial resolution ALOS panchromatic image was segmented by multi-resolution segmentation method. Second, the spectral features of segmented regions were extracted from multi-spectral ALOS image and TM image by spatial mapping mechanism. Third, the regions were classified by SVM classifier. Experimental results show that the classification method for high spatial resolution remote sensing images combining the TM spectral information based on the spatial mapping mechanism can make use of the spectral information both in high and low spatial resolution remote sensing images and improve classification accuracy.
\end{abstract}

\section{INTRODUCTION}

With the development of remote sensing technology, high spatial resolution remote sensing data have been more easily acquired and widely applied (Yuan and He, 2008). High spatial resolution remote sensing images, such as SPOT5, ALOS, ZY-3, Worldview, IKONOS and Quick Bird, have been applied for urban planning, urban change detection, ecological environment monitor, etc. The traditional method of classification for high spatial resolution images has been proven to have several drawbacks, such as low classification accuracy, the derivation of very limited spatial information, and salt and pepper effects (Chen et al., 2012). Therefore, novel and efficient analysis techniques are needed for processing and analyzing of high spatial resolution remote sensing images.

Object-based image analysis processing images at the object level instead of the pixel level is very effective for high spatial resolution remote sensing image classification. Many studies have been done on classification of high spatial resolution remote sensing images. Chen et al. (2012) introduced a modified object-oriented classification algorithm integrating multi-characteristics of high spatial resolution remote sensing image. Salehi et al. (2012) developed a hierarchical rule-based object-based classification framework coupled with height points for complex urban environment classification. Tang and Wang (2012) proposed an innovative approach for urban landuse and land-cover classification using marker-controlled watershed segmentation in the Vegetation-Impervious SurfaceSoil feature space. Brink and Eva (2011) analyzed the potential of TM and ETM data for land cover change detection. Zhang and Zhu (2011) put forward a knowledge-rule-based classification method for high spatial resolution remote sensing images combining the spectral, texture and shape features together. Although some improved algorithms can increase the classification accuracy by making use of spectral and textural information, the classification results still cannot satisfy all actual needs.

High spatial resolution remote sensing images contain more spatial information, and have increased the detail at which earth observations can be made. High spatial resolution remote sensing data often contain two types of images, those with a single panchromatic band and those with four multispectral bands(Wang et al., 2012). For example, ALOS images have a panchromatic band with a resolution of $2.5 \mathrm{~m}$ and four multispectral bands with a resolution of $10 \mathrm{~m}$. However, the high spatial resolution remote sensing images have less multispectral bands than low or medium spatial resolution remote sensing images. Take the TM images for example, TM images have six multispectral bands with a resolution of $30 \mathrm{~m}$ and one thermal infrared band with a resolution of $60 \mathrm{~m}$. The low or medium spatial resolution remote sensing images contain more spectral information than high spatial resolution remote sensing data.

In order to improve the accuracy of high spatial resolution remote sensing image classification, additional information should be incorporated into the classification process. This paper proposed a method of object-based land cover classification for high spatial resolution ALOS images combining the spectral information mapped from TM images. First, the high spatial resolution ALOS panchromatic image was segmented by multi-scale segmentation method. Second, the spectral features of segmented regions were extracted from multi-spectral ALOS image and TM image by spatial mapping mechanism. Third, the regions were classified by SVM classifier. The object-based SVM classification only applying

\footnotetext{
* Corresponding author. This is useful to know for communication with the appropriate person in cases with more than one author.
} 
on high spatial resolution ALOS images was used for comparison and the experimental results show that the classification method for high spatial resolution remote sensing images combining the TM spectral information based on the spatial mapping mechanism can make use of both high spatial resolution remote sensing image spatial information and low or medium resolution remote sensing image spectral information and can improve classification accuracy.

\section{METHODOLOGY}

The proposed method has three major steps. This section details the multi-scale image segmentation, the spectral feature extraction of segmented regions based on spatial mapping mechanism and Support Vector Machine classification. A flow chart of the proposed classification method is shown in Fig. 1.

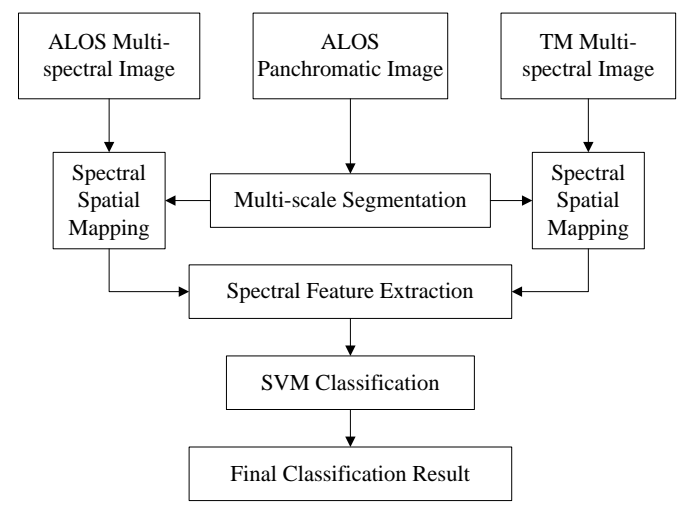

Figure 1 . The flow chart of the proposed classification method

\subsection{Multi-scale Image Segmentation}

The first step in the proposed method is the segmentation of panchromatic image. Image segmentation is an important part of image interpretation, especially for high spatial resolution remote sensing images(Zheng et al., 2013). High spatial resolution remote sensing images contain more information of ground objects, and show great diversity of them. The purpose of segmentation is to divide an image into homogeneous regions. In this paper, the region-based multi-resolution segmentation method available in eCogniton software was used. The segmentation process is controlled by the scale, shape and compactness parameters specified by users. For this study, the most suitable parameters for high spatial resolution ALOS panchromatic images were determined by experimenting and users' subjective assessment.

\subsection{Region Spectral Feature Extraction}

The second step in the proposed method is the spectral feature extraction of segmented regions. The regional spectral vector is calculated by the mean spectral vector of pixels contained in each region. The spectral vector of each pixel in a panchromatic image is obtained from ALOS and TM multispectral images by a spatial mapping mechanism.

The high spatial resolution remote sensing images have more spatial information. The low or medium resolution remote sensing images have more spectral information. In order to make full use of the information of both high and medium or low spatial resolution remote sensing image, image fusion is widely used to integrate the two together. The fusion algorithm merges the high resolution panchromatic and low resolution multispectral imagery to create an enhanced high resolution multispectral image. Then, the fused image is used in subsequent applications. Therefore, the fused image is an estimation which may cause spectral distortion and affect the accuracy of classification results(Wang et al., 2012). The effect of fusion directly determines the accuracy of subsequent applications.

In the proposed classification method, the pixel spatial relationship between high and low spatial resolution remote sensing images was fully considered. The low spatial resolution multispectral pixels were mapped to the segmentation results of high spatial resolution remote sensing images based on the spatial mapping mechanism. Taking a resolution ratio of 1:4 for example. One pixel in the high spatial resolution image corresponds to sixteen pixels in the low spatial resolution image. For example, if a pixel has position $\left(x_{i}, y_{j}\right)$ in the high spatial resolution image, then $i=1,2, \cdots, m ; j=1,2, \cdots, n ;$ the corresponding position of the pixel in the low spatial resolution image is $\left(x_{i}^{\prime}, y_{j}^{\prime}\right)$, where $x_{i}^{\prime}=\operatorname{Int}\left(x_{i} / 4+0.4\right)$ and $y_{j}^{\prime}=\operatorname{Int}\left(y_{j} / 4+0.4\right)$. The $\operatorname{Int}()$ function rounds a number to the nearest integer.

\subsection{Support Vector Machine Classification}

There are many image classification algorithms for remote sensing images. Each algorithm has its unique advantages and weaknesses. Support Vector Machine (SVM) is a supervised non-parametric statistical learning technique and widely used in classification of remote sensing images (Mountrakis et al., 2011). SVM is well at solving non-linear, high dimensional and limited training samples (Mountrakis et al., 2011). The SVM classifier with Gaussian radial basis function (RBF) kernel was applied in this paper. The optimal parameters $C$ (parameter that controls the amount of penalty during the SVM optimization) and $\gamma$ (parameter that describes the spread of the RBF kernel) were chosen by fivefold cross validation(Chang and Lin, 2011). LIBSVM library is used to implement the SVM classification algorithm(Chang and Lin, 2011).

\section{EXPERIMENT RESULTS AND ANALYSIS}

To evaluate the performance of the proposed method, two subsets of ALOS and TM images were used in a series of experiments and comparative analyses. To analyze the advantages of the proposed method for high spatial resolution remote sensing image classification, object-based SVM only on high spatial resolution ALOS fusion images was used for comparison. The panchromatic and multispectral images were fused by the PANSHARP method in PCI software. The regional spectral vector is calculated by the mean spectral vector of pixels contained in each region from high spatial resolution ALOS fusion images.

To assess classification accuracy, confusion matrices are obtained by selecting points with stratified random sampling. The reference classification images were generated through a precise manual interpretation on fusion images. The producer, user and overall classification accuracies are calculated from the confusion matrix.

ALOS images have a panchromatic band with a resolution of $2.5 \mathrm{~m}$ and four multispectral bands with a resolution of $10 \mathrm{~m}$. TM images have six multispectral bands with a resolution of 30 $\mathrm{m}$. In the first dataset, the size of ALOS multispectral image is $300 \times 300$, whereas that of the panchromatic image is $1200 \times 1200$. And the size of TM multispectral image is $100 \times 100$. The original images of ALOS and TM are shown in Fig. 2.The results of classification are shown in Fig. 3. Table 1 shows the 
producer, user and overall classification accuracies for the first ALOS and TM dataset classification. In the second dataset, the size of ALOS multispectral image is $900 \times 900$, whereas that of the panchromatic image is $3600 \times 3600$. And the size of TM multispectral image is $300 \times 300$. The original images of ALOS and TM are shown in Fig. 4.The results of classification are shown in Fig. 5. Table 2 shows the producer, user and overall classification accuracies for the second ALOS and TM dataset classification.
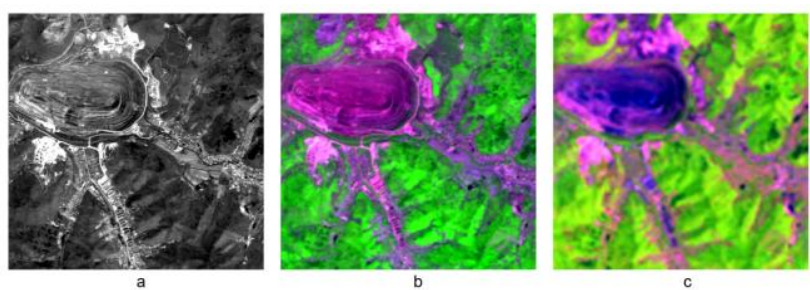

Figure 2. The ALOS and TM original image of the first dataset.

(a) is original image of ALOS panchromatic band, (b) is the synthetic image of ALOS 342 multispectral bands, and (c) is the synthetic image of TM 543 multispectral bands.
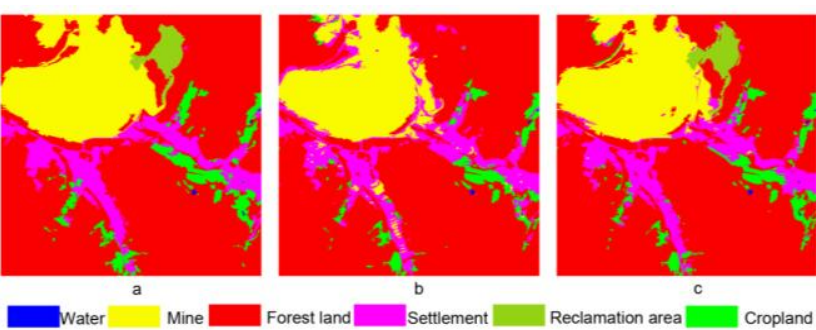

Figure 3. The classification results of the first dataset. (a) is the reference classification result generated through a precise manual interpretation on ALOS fusion images, (b) is the classification result only using ALOS image, and (c) is the classification result combining the ALOS and TM images.
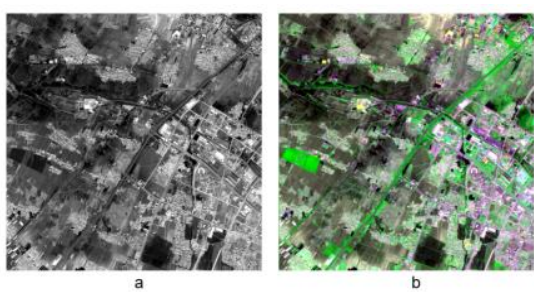

Figure 4. The ALOS and TM original image of the second dataset. (a) is original image of ALOS panchromatic band, (b) is the synthetic image of ALOS multispectral 342 bands, and (c) is the synthetic image of TM 543 multispectral bands.
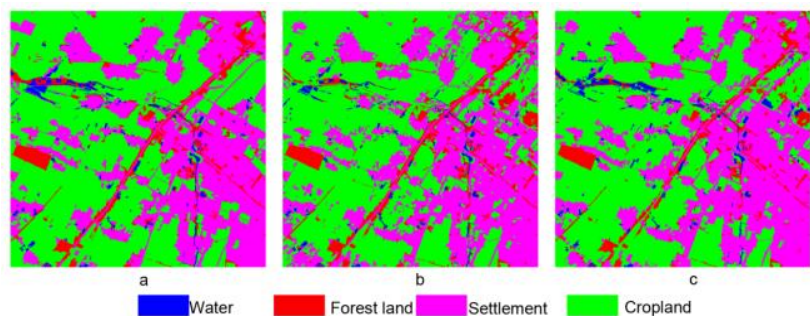

Figure 5. The classification results of the second dataset. (a) is the reference classification result generated through a precise manual interpretation on ALOS fusion images, (b) is the classification result only using ALOS image, and (c) is the classification result combining the ALOS and TM images.

\begin{tabular}{|c|cc|cc|}
\hline Class & \multicolumn{2}{|c|}{ The proposed method } & \multicolumn{2}{|c|}{$\begin{array}{r}\text { Object-based SVM Only } \\
\text { ALOS }\end{array}$} \\
\hline & User's & Producer's & User's & Producer's \\
Accuracy & Accuracy & Accuracy & Accuracy \\
Water & 100.00 & 100.00 & 83.33 & 100.00 \\
Reclamation & 87.27 & 82.76 & 0.00 & 0.00 \\
Mine & 94.62 & 97.23 & 84.62 & 87.88 \\
Forest land & 97.30 & 94.85 & 96.56 & 93.35 \\
Settlement & 88.26 & 84.74 & 79.20 & 69.40 \\
Cropland & 64.17 & 80.69 & 53.15 & 71.81 \\
\hline Average & \multirow{2}{*}{88.60} & 90.05 & 66.14 & 70.41 \\
accuracy & \multicolumn{2}{|c|}{92.25} & \multicolumn{2}{|c}{85.54} \\
\hline Overall & \multicolumn{3}{|c}{} \\
accuracy & \multicolumn{2}{|c}{} \\
\hline
\end{tabular}

Table 1. Producer, user and overall classification accuracies for the first dataset

\begin{tabular}{|c|c|c|c|c|}
\hline Class & \multicolumn{2}{|c|}{ The proposed method } & \multicolumn{2}{|c|}{$\begin{array}{c}\text { Object-based SVM Only } \\
\text { ALOS }\end{array}$} \\
\hline Water & $\begin{array}{c}\text { User's } \\
\text { Accuracy } \\
71.00\end{array}$ & $\begin{array}{c}\text { Producer's } \\
\text { Accuracy } \\
75.37\end{array}$ & $\begin{array}{c}\text { User's } \\
\text { Accuracy } \\
68.45\end{array}$ & $\begin{array}{c}\text { Producer's } \\
\text { Accuracy } \\
83.81\end{array}$ \\
\hline $\begin{array}{l}\text { Forest } \\
\text { land }\end{array}$ & 82.39 & 79.24 & 81.03 & 75.54 \\
\hline Settlement & 92.84 & 90.21 & 89.48 & 89.25 \\
\hline Cropland & 86.13 & 88.18 & 84.53 & 84.70 \\
\hline $\begin{array}{l}\text { Average } \\
\text { accuracy }\end{array}$ & 83.09 & 83.25 & 80.87 & 83.33 \\
\hline $\begin{array}{l}\text { Overall } \\
\text { accuracy }\end{array}$ & \multicolumn{2}{|c|}{88.54} & \multicolumn{2}{|c|}{86.54} \\
\hline
\end{tabular}

Table 2. Producer, user and overall classification accuracies for the second dataset

By comparing the accuracies of the classification results, the proposed object-based method combining TM spectral information based on a spatial mapping mechanism can be seen to obtain better classification results than object-based SVM only using high spatial resolution remote sensing images. Table 1 and 2 show that the overall accuracies of the proposed method are higher than object-based SVM only using high spatial resolution remote sensing images.

According to the experimental results, the proposed classification method combing TM spectral information based on a spatial mapping mechanism can obtain higher accuracy than the object-based SVM only using high spatial resolution remote sensing images. The proposed method can make full use of both the spatial information in high and the spectral information in low spatial resolution remote sensing images.

In this paper, only spectral features in the images were applied to the classification process. Further work is required to integrate the textural and shape features of high spatial remote sensing images.

\section{CONCLUSIONS}

A new object-based high spatial resolution remote sensing image classification method combining the TM spectral information has been presented in this paper, in which the spectral information of low spatial resolution remote sensing images was integrated by a spatial mapping mechanism. Furthermore, the proposed method was applied on raw remote 
sensing data instead of fusion images. Experimental results have demonstrated that the proposed method can make full use of the information in both high and low spatial resolution remote sensing images and obtain higher final classification accuracy.

\section{ACKNOWLEDGMENTS}

This work was sponsored by the National Natural Science Foundation of China under the grant number 60972142. The authors wish to thank the anonymous reviewers who provided constructive comments that improved the quality and clarity of the manuscript.

\section{REFERENCES}

Brink, A. B., Eva, H. D., 2010. The potential use of highresolution Landsat satellite data for detecting land-cover change in the Greater Horn of Africa. International Journal of Remote Sensing, 32:21, pp. 5981-5995.

Chang, C. C., Lin, C. J., 2011. LIBSVM: a library for support vector machines. ACM Transactions on Intelligent Systems and Technology, 2(27), pp. 1-27.

Chen, Z., Wang, G., Liu, J., 2012. A modified object-oriented classification algorithm and its application in high-resolution remote-sensing imagery. International Journal of Remote Sensing, 33(10), pp. 3048-3062.

Mountrakis, G., Im, J., Ogole, C., 2011. Support vector machines in remote sensing: A review. ISPRS Journal of Photogrammetry and Remote Sensing, 66, pp. 247-259.

Salehi, B., Zhang, Y., Zhong, M., Dey, V., 2012. Object-Based Classification of Urban Areas Using VHR Imagery and Height Points Ancillary Data. Remote Sensing, 4, pp. 2256-2276.

Tang, Q., Wang, L., 2012. Urban land-use, land-cover classification through watershed segmentation in the V-I-S feature space. Remote Sensing Letters, 3:8, pp. 677-685.

Wang, G. Z., He, G. J., Liu, J. B., 2012. A new classification method for high spatial resolution remote sensing image based on mapping mechanism. In: The 4th International Conference on Geographic Object-Based Image Analysis, Rio de Janeiro, Brazil, pp.186-190.

Yuan, J. Y., He, G. J., 2008. A new classification algorithm for high spatial resolution remote sensing data. In: The International Conference on Earth Observation Data Processing and Analysis, Wuhan, China, v.7285.

Zhang, R., Zhu, D., 2011. Study of land cover classification based on knowledge rules using high-resolution remote sensing images. Expert Systems with Applications, 38, pp. 3647-3652.

Zheng, C., Wang, L., Chen, R., Chen, X., 2013. Image Segmentation Using Multiregion-Resolution MRF Model. IEEE Geoscience and Remote Sensing Letters, 10(4), pp. 816-820.

Revised August 2013 